\title{
A genome-wide search for new imprinted genes in the human placenta identifies DSCAM as the first imprinted gene on chromosome 21
}

\author{
Laïla Allach El Khattabi ${ }^{1,2,3,4} \cdot$ Stéphanie Backer ${ }^{1,2,3} \cdot$ Amélie Pinard $^{1,2,3,8} \cdot$ Marie-Noëlle Dieudonné $^{5}$. \\ Vassilis Tsatsaris ${ }^{6}$ - Daniel Vaiman $\mathbb{1}^{1,2,3} \cdot$ Luisa Dandolo $^{1,2,3} \cdot$ Evelyne Bloch-Gallego ${ }^{1,2,3} \cdot$ Hélène Jammes $^{7}$. \\ Sandrine Barbaux (iD) $1,2,3$
}

Received: 18 December 2017 / Revised: 16 July 2018 / Accepted: 23 August 2018 / Published online: 11 September 2018

(c) European Society of Human Genetics 2018

\begin{abstract}
We identified, through a genome-wide search for new imprinted genes in the human placenta, DSCAM (Down Syndrome Cellular Adhesion Molecule) as a paternally expressed imprinted gene. Our work revealed the presence of a Differentially Methylated Region (DMR), located within intron 1 that might regulate the imprinting in the region. This DMR showed a maternal allele methylation, compatible with its paternal expression. We showed that DSCAM is present in endothelial cells and the syncytiotrophoblast layer of the human placenta. In mouse, Dscam expression is biallelic in foetal brain and placenta excluding any possible imprinting in these tissues. This gene encodes a cellular adhesion molecule mainly known for its role in neurone development but its function in the placenta remains unclear. We report here the first imprinted gene located on human chromosome 21 with potential clinical implications.
\end{abstract}

\section{Introduction}

Imprinting is a complex heritable epigenetic regulation of gene expression resulting in a monoallelic expression of a

Electronic supplementary material The online version of this article (https://doi.org/10.1038/s41431-018-0267-3) contains supplementary material, which is available to authorized users.

Sandrine Barbaux

sandrine.barbaux@inserm.fr

INSERM, U1016, Institut Cochin, Paris, France

2 CNRS UMR8104, Paris, France

3 Université Paris Descartes, Sorbonne Paris Cité, Paris, France

4 Laboratoire de Cytogénétique, Hôpital Cochin, AP-HP, Paris, France

5 Université Versailles-Saint Quentin en Yvelines, Versailles, France

6 Maternité Cochin-Port Royal, AP-HP, Paris, France

7 INRA, UMR1198 Biologie du Développement et de la Reproduction, Jouy en Josas, France

8 Present address: Division of Medical Genetics, McGovern Medical School at The University of Texas Health Science Center, Houston, USA set of genes according to the parent-of-origin of the allele. These genes belong to various families including growth factors, transcription factors, enzymes, receptors, and are involved in placental and foetal development. This is supported by the fact that (i) nuclear transfer experiments leading to gynogenote or androgenote embryos result in lethal abnormal foetal or placental development [1], (ii) abnormal imprinting of some imprinted genes is associated with placental and foetal growth pathologies such as preeclampsia and intra-uterine growth restriction [2, 3], (iii) imprinting is extensive during human development and bares, mainly, a placental-specific pattern [4], and (iv) placenta and imprinting appearance are concomitant in terms of evolution [5].

About 150 imprinted genes (IGs) are known in mice and close to 100 in humans. Some of them have been identified following the molecular characterisation of chromosomal rearrangements or uniparental disomies causing clinical syndromes (Prader-Willi syndrome and Beckwith-Wiedemann syndrome, for instance). IGs are interesting for their particular epigenetic regulation and their implication in foeto-placental development with a role in foeto-maternal nutrient exchanges, brain development as well as in behaviour $[6,7]$. Diverse strategies have been adopted to identify new candidate imprinted genes in humans and mice. 
These were, mainly and not exclusively, based on monoallelic gene expression pattern [8], differential allelic methylation [9, 10], using placental tissue [11], parthenogenetic tissues [2, 12] or triploidies [4, 9]. Other studies, based on computational predictions [13, 14], used putative genomic specificities such as gene organisation or presence of repeated elements to provide lists of candidate genes. However, validation approaches to confirm the imprinted status of these predicted genes still did not unveil all IGs and many discrepancies exist between results emanating from different studies and from different teams. To identify new genes that could be imprinted or at least have a monoallelic expression profile in the human placenta, we designed an efficient strategy using high throughput genotyping arrays. A first validation step allowed the characterisation of 7 candidates as new IGs in the human placenta, one of them being shown as also imprinted in mouse [15]. Here, we report the results of an extended analysis of our previous data, exploring 30 candidate genes and leading to the discovery of the first imprinted gene located on human chromosome 21, Down Syndrome Cellular Adhesion Molecule or DSCAM, among other candidates.

\section{Materials and methods}

\section{Biological human samples}

Fifty human placentas from pathologic (Intra-Uterine Growth Restriction (IUGR), preeclampsia) or normal pregnancies as well as most matched maternal peripheral blood were collected at the Port Royal-Cochin Obstetrics and Gynaecology Department (Paris, France). Placenta samples were treated within $30 \mathrm{~min}$ after delivery by caesarean section, dissected to remove the maternal side and snap frozen before further extraction. White blood cells were isolated and frozen for DNA extraction as previously described [16]. Clinical details have already been described [17].

Sperm samples were obtained from normozoospermic men attending the Cochin Hospital infertility clinic. After a density gradient, pellets of sperm were collected to extract genomic DNA.

\section{Mouse tissues}

Mus musculus molossinus and C57/B6 mice were mated. F1 foetal tissues (hindbrain, cerebellum, spinal cord, midbrain, cortex, whole placenta, and labyrinth/junctional zone placental sections) were collected at E13.5, E15.5, E16.5, or E18.5 from 10 reciprocal crosses.

\section{Primary cells}

Primary cytotrophoblasts were isolated as follows: first trimester human placental tissues from pregnancy abortions were placed in saline $(\mathrm{NaCl} 150 \mathrm{mM})$, washed several times, and dissected to remove decidual tissues and foetal membranes. Cytotrophoblasts (CTs) were isolated from tips of placental villi as previously described [18]. The purity of isolated CTs exceeds 95\% as assessed by cytokeratin-7 expression using immunocytochemistry. Moreover, purified CTs were characterised by (i) the observation of cell aggregates and syncytiotrophoblast from $48 \mathrm{~h}$ to $72 \mathrm{~h}$ time of culture, and (ii) the measures of hCG secretion in culture medium after 24,48 , and $72 \mathrm{~h}$ of cell culture [19].

\section{Cell culture}

The Human Umbilical Vein Endothelial Cell (HUVEC) cell line and the monkey kidney fibroblast COS-7 cell line were grown in DMEM medium while the human choriocarcinoma cell line BeWo was cultured in F12K medium, both supplemented with penicillin/streptomycin (plus $0.1 \%$ Normocin for HUVECs) and $10 \%$ Foetal Calf Serum, at $37^{\circ}$ $\mathrm{C}$ and $5 \% \mathrm{CO}_{2}$. BeWo cells were treated with $12 \mu \mathrm{M}$ Forskolin for $48 \mathrm{~h}$ to induce syncytialisation. Transfections of COS-7 cells in 6-well plates were performed using 10 or $15 \mu \mathrm{l}$ of the Lipofectamine 2000 reagent (Life Technologies, USA) and $14 \mu \mathrm{g}$ of a human pcDNA3.1 + /DSCAMC-DYK expressing vector obtained from GenScript, Piscataway, USA. Cellular extracts were harvested $48 \mathrm{~h}$ after transfection.

\section{Nucleic acids extraction and reverse transcription}

Genomic DNA was extracted from cells or tissues according to [16]. RNA was extracted for human placental tissues or from animal samples using Trizol (Life Technologies, St Aubin, France) followed by chloroform extraction and precipitation. Concentration and purity were tested on a Nanodrop spectrophotometer (Nanodrop, Thermo Scientific, Illkirsch, France). Two micrograms of RNA were treated with DNase in order to eliminate potential DNA residues before Reverse Transcription. RNA was then exposed to MMLV Reverse Transcriptase in the presence of hexamer random primers (Thermo Scientific, Illkirsch, France).

\section{Strategy of identification of candidate imprinted genes}

We used data from our previously described work based on a whole genome screening strategy using genotyping 
microarrays [15]. Briefly, this consisted in hybridising, in parallel, pairs of $250 \mathrm{~K}$ genotyping Affymetrix arrays with either genomic DNA or cDNA extracted from five human placentas. Generated fluorescence data were analysed using the Affymetrix algorithm [15], Manufacturer's thresholds (default settings) for allele call were applied.

Called alleles in genomic DNA and their matched cDNA were then used to identify informative SNPs (heterozygous) from genomic DNA for which we analysed the allelic expression based on the cDNA genotyping data (summarised in Supplemental Fig. 1). Genes containing heterozygous SNPs that were found to be monoallelic in cDNA were considered as potential candidate genes with monoallelic expression. We established a pipeline to select a list of candidate monoallelic/imprinted genes as we previously described [15]. Here, we extended our analysis to an additional list of candidate genes suspected to have a monoallelic expression. After a positive hit on chromosome 21, we specifically reanalysed data from our original arrays for this chromosome using a less stringent filter on the quality score of genotyping results calculated by Affymetrix algorithm $(0.1$ instead of 0.05$)$ to extract more candidates.

\section{Validation of candidate imprinted genes}

Candidate genes identified from microarray genotyping analysis were verified by Sanger sequencing of genomic DNA and their matched cDNA from a larger set of new placenta samples $(n=39)$. For each gene, we analysed at least one SNP selected to be (i) exonic and (ii) found heterozygous in genomic DNA. Subsequently, the candidate gene was confirmed or not to have a monoallelic expression.

When monoallelic expression was confirmed, we investigated the parental origin of the expressed or silenced allele by genotyping maternal blood DNA when available.

We validated this approach by performing a pyrosequencing of one of our newly identified IGs to quantify the allelic ratio.

\section{Identification of differentially methylated regions}

For one gene, we comprehensively validated the imprinted status and studied the methylation level of relevant $\mathrm{CpG}$ islands within and around the gene in order to identify a Differentially Methylated Region (DMR) typical of an imprinted gene. We first identified $\mathrm{CpG}$ islands in silico using the CpGplot software. We selected the most relevant sites according to their location in the gene (promoter regions) and performed a methylation level analysis as follows: Genomic DNA was treated with sodium bisulfite in order to modify unmethylated cytosines using the EZ DNA Methylation-Gold Kit (Zymo Research, Irvine, CA). Eighty nanograms of bisulfite converted DNA were used as template for PCR amplification in classical conditions. PCR products were either directly sequenced at the Eurofins Cochin platform after purification using the MachereyNagel kit, or used as templates for pyrosequencing reactions, or cloned using the TOPO TA cloning kit (Thermofischer) and sequenced.

\section{Pyrosequencing studies}

Pyrosequencing was performed using the PyroMark 24 Gold Kit on a PyroMark Q24 System (Qiagen, Courtaboeuf, France) according to the manufacturer's recommendations. Duplicate measures were obtained on different PCR products amplified on bisulfite-treated gDNA samples performed on 2 occasions. A difference of more than $5 \%$ between duplicates was considered uncertain and repeated. Genomic DNAs from 25 placental samples and 6 sperm samples were analysed. Pyrosequencing was also performed on cDNA samples around rs34336407 to quantify the allelic ratio. Primer sequences and applications are listed in Supplemental Table 3.

\section{PCR and real-time PCR}

Amplifications were performed using the GoTaq Flexi or HotStart GoTaq Flexi (Promega, Charbonnières, France) following the manufacturer's conditions. Real-time RTPCR was performed on a LightCycler480 thermocycler (Roche, Mannheim, Germany) using the LightCycler480 $\mathrm{SYBr}$ Greeen I Master Mix (Roche). We used three reference genes that have been previously recommended for the analysis of placental tissue (SDHA, TBP and YWHAZ) [20]. Primers, applications, and particular conditions are described in Supplemental Table 3. Results were analysed following the $\Delta \Delta \mathrm{Ct}$ method. Statistical analysis was performed using a Post Hoc test, namely the Student-NewmanKeuls test, for multiple comparisons. Results were considered statistically significant when the $p$-value was below 0.05 .

\section{STR analysis}

For one sample, we performed a DNA genotyping using the PowerPlex $^{\circledR} 16$ HS multiplex STR System (Promega) to exclude a sample swap. The analysis was performed on $2 \mathrm{ng}$ of placental genomic DNA, $750 \mathrm{ng}$ of corresponding placental RNA and 2 ng of matched maternal gDNA. After loci co-amplification, microsatellite repeat alleles were analysed by a standard semiautomatic method on an ABI PRISM 377 automate (Applied Biosystems, Thermofischer). Genotyping data were analysed using the Gene Mapper version 3.5 software package (Applied Biosystems). 


\section{Immunohistochemistry and western blot}

Paraffin-embedded placental sections from normal term pregnancies or from earlier terminated pregnancies were rehydrated and boiled in citrate buffer. They were treated to block endogenous peroxidases with hydrogen peroxide and unspecific binding sites with BSA. The primary antibody NBP1-59208 against DSCAM (Biotechne, Lille, France) was incubated in PBS- $0.1 \%$ Tween- $1 \%$ BSA overnight at $4{ }^{\circ} \mathrm{C}$. After washing, revelation was obtained using the Novolink kit (Leica Biosystems, Nanterre, France).

Ten to $40 \mu \mathrm{g}$ of protein lysate from BeWo, HUVEC cells or COS-7 transfected cells were run on a $10 \%$ polyacrylamide gel and transferred to a PVDF membrane. Revelation was performed using the anti-DSCAM antibody or an anti-tubulin (05-661 from Millipore, Molesheim, France), an anti-rabbit secondary antibody and the Immobilon kit (Millipore).

\section{Ethical considerations}

Patients have been informed about the study in a dedicated visit and have given their informed consent to collect and use their biological samples. All protocols have been approved by the local Ethics Committee (No CPP Am57241-COL2991; CODECOH No DC-2012-1645).

\section{Results}

\section{Identification and validation of candidate imprinted genes}

Based on our microarray studies, we identified a list of 30 genes (Supplemental Table 1). After the validation process, 16 genes were found to have a biallelic expression, 11 were inconclusive for either technical issues or insufficient/no heterozygous individuals (rare SNPs), 2 were confirmed to be imprinted and one showed results supporting his imprinted status though some questions remain regarding the parental origin of the expressed allele.

We analysed the AIMI (Absent in Melanoma 1) gene, also known as CRYBG1, on chromosome 6 using the rs11152999:G > A SNP (NC_000006.12:g.106512572 G > A) at the beginning of the ORF and could identify two heterozygous placentas exhibiting a monoallelic expression. Given the weak frequency of this polymorphism in Caucasian populations, we analysed another SNP, namely rs2297970:G > A (NC_000006.12:g.106551947 G > A), located around the middle of the ORF. We obtained conflicting results consisting in two cases of placental monoallelic expression but also six cases of biallelic expression using this SNP. We observed that the first SNP is present only in the long isoform of the gene while the second is located in an exon common to both the long and short isoforms, the 2 isoforms being the result of alternative promoter usage. This result has also been described by Das et al. [21] and confirms that the imprinting of AIMI is isoform-specific and limited to the longest transcript that uses a different upstream promoter.

To analyse the NCAM2 (Neural Cellular Adhesion Molecule 2) gene on chromosome 21, we used 2 SNPs, rs232518:T > C (NC_000021.9:g.21373867 T > C) in exon 9 and rs2017705:A > G (NC_000021.9:g.21508930 A > G) in exon 16 (Supplemental Table 2) [22]. Most of the heterozygous placentas showed a monoallelic expression $(n=$ 15 ) and in the four informative cases, where the mother was heterozygous allowing for tracing the origin of the expressed allele, this later was of maternal origin. However, one case showed a biallelic expression, on repeated experiments though the position of cDNA specific primers could not allow the amplification of potentially contaminating gDNA. Such polymorphic imprinting has already been described for other imprinted genes [23]. Another conflicting case is one showing a monoallelic expression but with a paternal origin of the expressed allele. To exclude a problem regarding the amplified samples, we used an analysis of Short Tandem Repeats to confirm that the RNA sample and the genomic DNA extracted were indeed from the same individual and that the maternal DNA matches with the child DNA. All three samples were correct (Supplemental Fig. 2). The exact status of the NCAM2 gene is therefore still under question.

For a few candidates, we could not confirm the monoallelic expression or could not conclude because of lack of informative SNPs, insufficient informative subjects or insufficient expression level (Supplemental Table 1). These genes remain good candidates to explore in more and larger studies.

We focused further on a promising candidate, namely DSCAM for Down Syndrome Cellular Adhesion Molecule, located on chromosome 21, in the 21q22 chromosomal band. We explored the imprinted status of this gene by investigating the parent-of-origin expression of two SNPs in exon 5 (exon numbering according to Agarwala et al. [24]), rs2297270:G > C (NC_000021.9:g.40353703 G > C) and rs41395652:G > A (NC_000021.9:g.40353698 C > T). We observed 10 cases of heterozygous carriers of these polymorphisms showing a monoallelic expression of the DSCAM gene in placental cDNA. By analysing a third SNP, rs34336407:G > A (NC_000021.9:g.40312163 C > T) in exon 9 of DSCAM, we found three additional heterozygotes who also exhibited a monoallelic expression in placental tissue. No case of biallelic expression was observed among all tested samples (Supplemental Table 2). In seven cases, 
A)

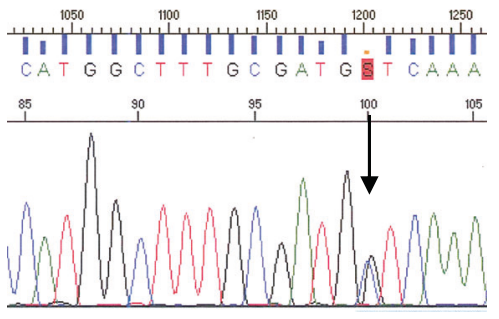

Placental gDNA

B)

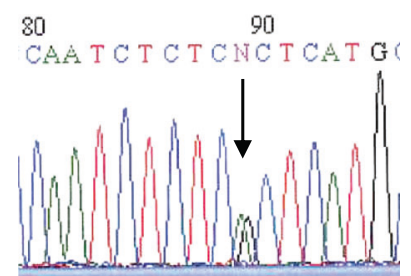

Placental gDNA

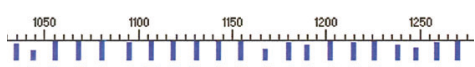

CAT G G C TTTGCGAT G GT CAAA

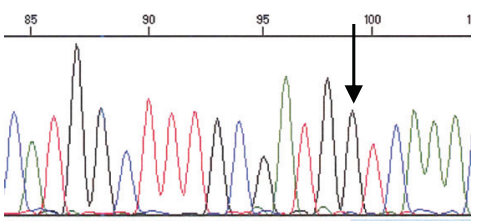

Placental cDNA

110

CAA T C T C T CAC T CA T G

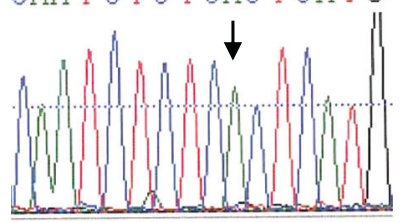

Placental cDNA
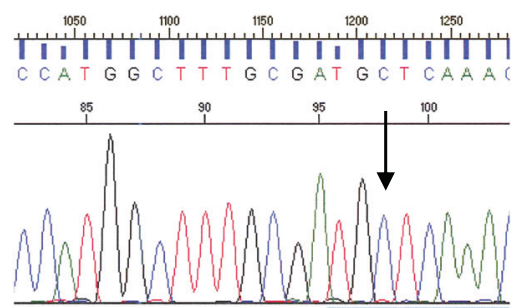

Maternal gDNA

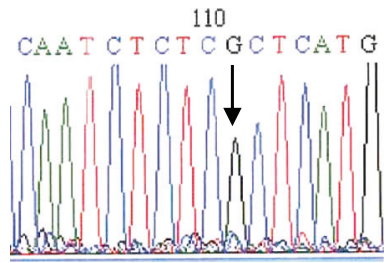

Maternal gDNA

C)

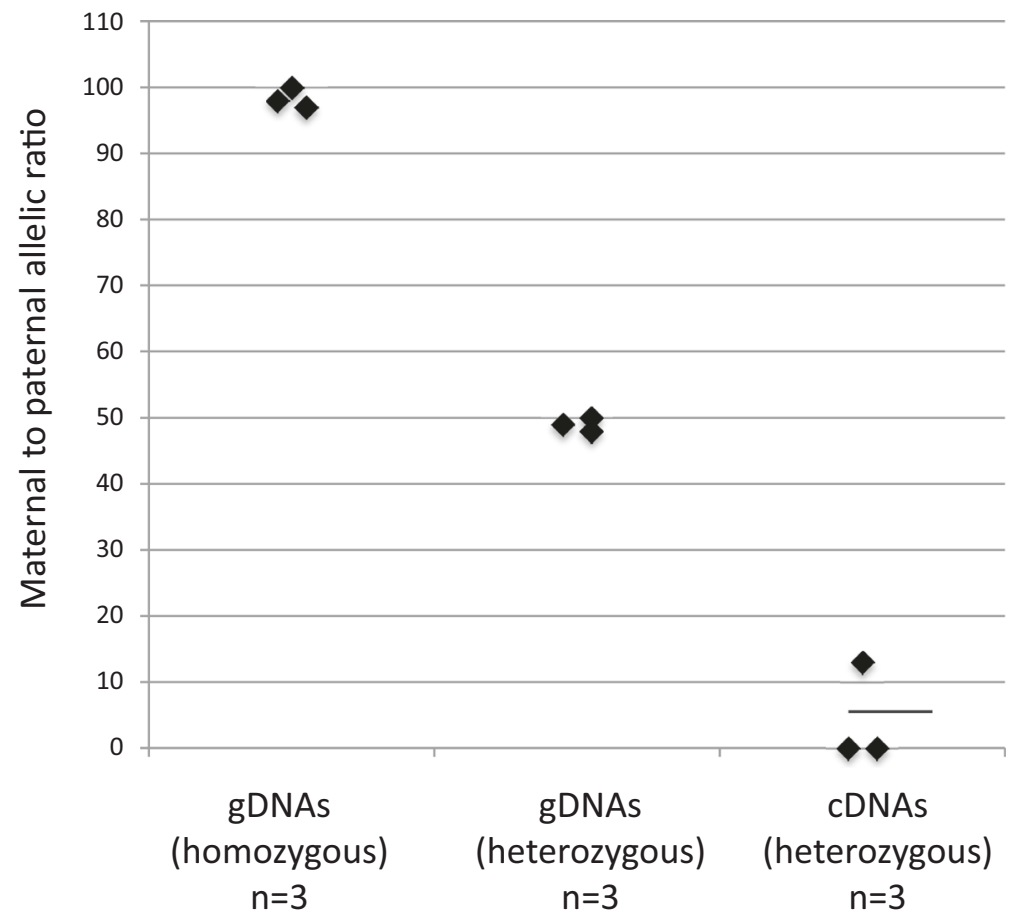

Fig. 1 Imprinting analysis of the $D S C A M$ gene. a, b Sequences from representative individuals' genomic DNAs, placental cDNAs and maternal genomic DNAs around polymorphisms (black arrows) rs41395652 in exon 5 (a) and rs34336407 in exon 9 (b). c Maternal to

the genotyping of maternal genomic DNA was informative to assess the parental transmission of the alleles. In all those cases (five cases for the two SNPs in exon 5 and two cases for the SNP in exon 9), the expressed allele was the paternal one (Fig. 1a, b). Supplemental Fig. 3 shows the whole series paternal allelic ratios as estimated by pyrosequencing for rs 34336407 . Control samples consisting in heterozygous and homozygous genomic DNAs were used to check the obtained vs. expected ratios (1 for homozygous and 0.5 for heterozygous samples)

of chromatograms where the second allele is completely absent or very limited. By pyrosequencing, we could quantify this ratio for rs34336407, the maternal allele represents only $5 \%$ of the cDNA expression, on average (Fig. 1c). 
Fig. 2 Epigenetic analysis of the DSCAM gene. a Map of the DSCAM locus on chromosome 21. Upper bands represent exons of the DSCAM (black), DSCAM$A S 1$ and DSCAM-IT1 (grey) genes. The grey curved arrows represent the transcription starts of DSCAM. CpG islands located within and around the gene are shown as stars. $\mathrm{CpG}$ island \#22bis (in grey) is detailed with each band representing a $\mathrm{CpG}$ dinucleotide (position 40841468-40842223 on chromosome 21 (Genome version hg38)). b Sequencing of the $\mathrm{CpG} \# 22$ bis island region from placental genomic DNA treated by bisulfite. c DNA methylation level of $\mathrm{CpG}$ island \#22bis by pyrosequencing. The grey squares represent the average of 25 placental samples, \pm standard deviation, while black triangles were data obtained from 6 sperm samples, \pm standard deviation. d DNA methylation level of $\mathrm{CpG}$ island \#22bis region by cloningsequencing from a representative placental sample. White dots represent unmethylated cytosines and black dots, methylated cytosines

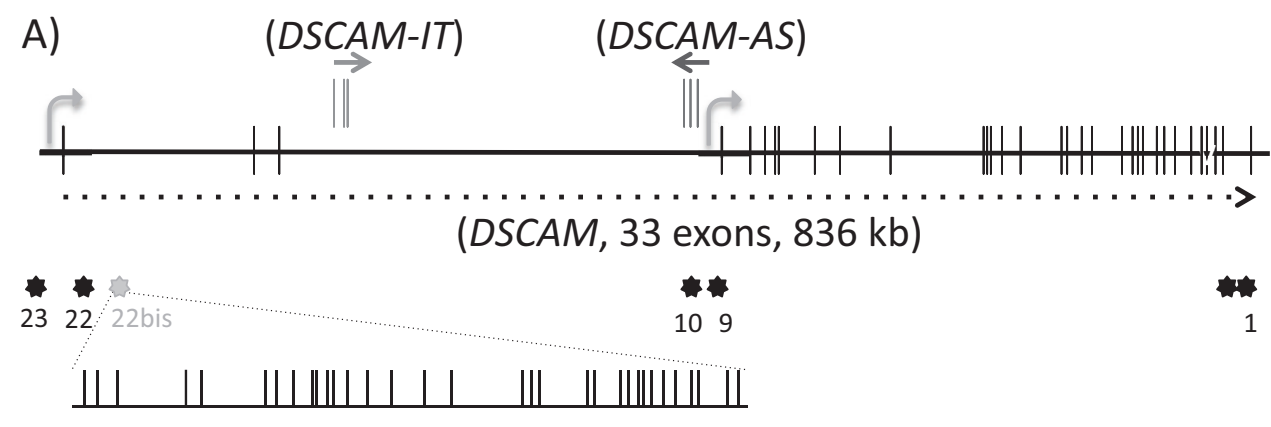

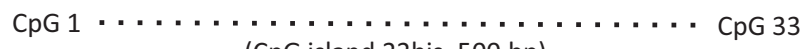

(CpG island 22bis, $500 \mathrm{bp}$ )

B)

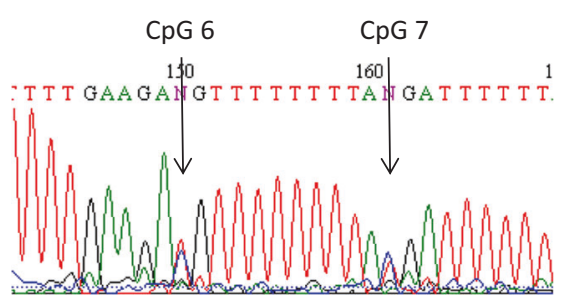

C)

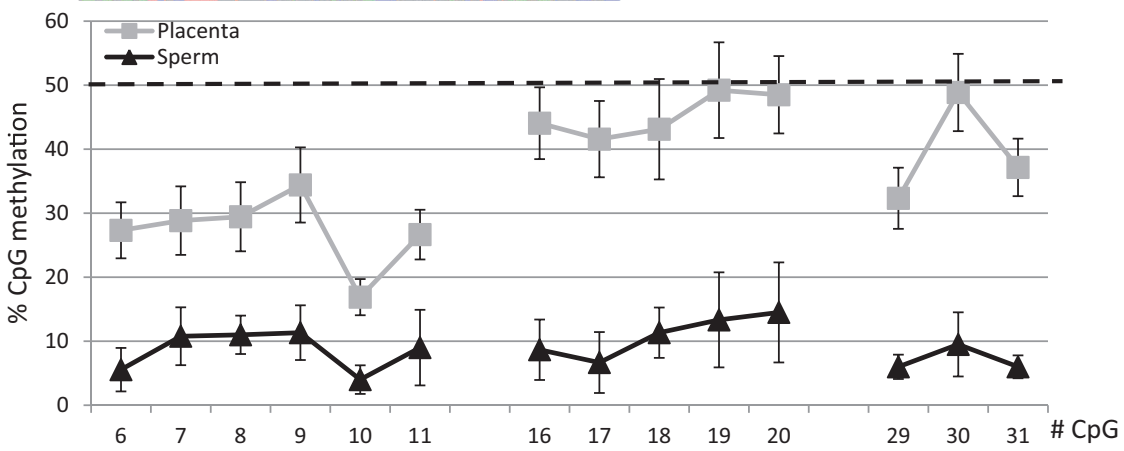

D)
We therefore concluded that DSCAM is imprinted in the human placenta. No expression could be detected in lymphocytes to extend the imprinting analysis.

At the DSCAM locus, an antisense gene (DSCAM$A S 1)$ composed of two or three exons is observed as well as a 4-exon intronic transcript (DSCAM-IT1) (Fig. 2a). Both overlap the very large intron 3 of DSCAM on opposite strands and are non-coding transcripts. Though we tried to determine the imprinted status of DSCAM-AS1 and DSCAM-IT1 using rs2837597:G > C (NC_000021.9:g.40383572 C > T) and rs2837753:T >C (NC_000021.9:g.40618823 A $>$ G) respectively, the expression of these genes was too weak in placental tissue and did not allow their amplification and further investigation.

\section{Epigenetic regulation of DSCAM imprinting}

We studied the methylation of $\mathrm{CpG}$ islands within and around DSCAM in order to identify a Differentially Methylated Region (DMR) typical of an imprinted gene. An in silico analysis of the genic sequence using the CpGplot software predicted the presence of $23 \mathrm{CpG}$ islands (Fig. 2, a). We limited our subsequent investigations to the most 
relevant sites. Hence, we first selected three predicted $\mathrm{CpG}$ islands for which we studied the methylation status, one in the promoter region of the long DSCAM isoform (named $\mathrm{CpG} 23$ ) and two (named $\mathrm{CpG} 9$ and $\mathrm{CpG} 10$ ) that locate, respectively, within the promoter region of the antisense gene, DSCAM-AS1, and the promoter region of the shorter DSCAM transcript. We first performed Sanger sequencing after bisulfite treatment of gDNA around these regions. We could not find any differential methylation of the CpG23, CpG9, and CpG10, these being mainly and globally methylated. A genome-wide search for DMR using methylation arrays had noted the presence of such a $500 \mathrm{bp}$ region about $20 \mathrm{~kb}$ from $\mathrm{CpG}$ island 22, within the very large intron 1 of the DSCAM gene and thereafter called \#22bis [25]. Direct sequencing after bisulfite treatment of placental DNA provided a double peak sequence typical of a DMR at this location (Fig. 2b). We performed a Pyrosequencing approach to precisely evaluate the methylation levels. In 25 placental genomic samples, the average methylation level ranges between $30-50 \%$ as often observed with imprinted genes, although variations are observed at different individual CpGs (Fig. 2c). We then cloned amplimers from this $\mathrm{CpG}$ \#22bis region and sequenced 10-20 clones in two representative individuals. This confirmed that unmethylated $\mathrm{CpG}$ dinucleotides tend to cluster on the same allele while methylated ones are also in phase on the other allele, suggesting a close to 50/50 allelic repartition (Fig. 2d). However, because of the presence of only 3 SNPs within the region, rare in the Caucasian population (rs79566420:A $>\mathrm{G} \quad$ (NC_000021.9:g.40842004 A > G), rs73227948: $\mathrm{G}>\mathrm{A}$ (NC_000021.9:g.40841821 G > A) and rs114498344: C > T (NC_000021.9:g.40841770 C > T), we were not able to identify heterozygous carriers in order to assess the parental origin of the hypomethylated and hypermethylated alleles. We therefore attempted to analyse the methylation level of this region in DNA extracted from six sperm samples, representing the paternal allele. In these cases, the methylation level is significantly low, at least $30 \%$ below that of placental DNA (Fig. 2c). This suggests that the methylated allele is the maternal one, consistent with a paternal expression of DSCAM. We therefore concluded that a DMR is present in intron 1 of the $D S C A M$ gene, with a methylation imprint on the maternal allele.

Another maternally methylated DMR has been described [26] about $600 \mathrm{~kb}$ from the DSCAM gene, near the WRB gene (Tryptophan Rich Basic protein). We checked the imprinted status of this gene and of PLAC4 (Placenta specific protein 4), located $300 \mathrm{~kb}$ from DSCAM and highly expressed in the placenta using rs35946782:G $>$ A (NC_000021.9:g.39391828 G > A), and rs9305729:C > G (NC_000021.9:g.41178475 C > G) respectively. But both appeared to have a biallelic expression in placenta, in consistency with others for WRB [26].

\section{DSCAM cellular expression}

In the literature, DSCAM expression is mainly described in neurons. Its function in the placenta was not yet investigated. We therefore analysed DSCAM expression in placental tissue by immunohistochemistry to identify which cell type expresses DSCAM. A positive and strong labelling was observed in endothelial cells of placental capillaries and in syncytiotrophoblasts. The same result was observed whatever the term from early (13 and 19 weeks of amenorrhea) to late term (30-40 weeks) placentas (Fig. 3). DSCAM immunolabelling appears mainly in the cytoplasmic compartment of positive cells. A diffuse labelling is seldom detected in mesenchymal cells. Positive signals disappear in the presence of a blocking DSCAM peptide but not in the presence of an irrelevant peptide. Similar observations were obtained on sections from IUGR affected pregnancies.

DSCAM expression was also validated by Western blot, using samples from cell line models: extracts from both the HUVEC endothelial cell line and the choriocarcinoma trophoblastic cell line BeWo, as well as from COS-7 cells transfected with a DSCAM-expression vector, revealed the expression of a $200 \mathrm{kDa}$ protein that confirms the specific expression of DSCAM and the specificity of the antibody (Supplemental Fig. 4).

\section{DSCAM expression in placental tissues}

As an imprinted gene expressed in the placenta, DSCAM becomes a candidate for a role in pregnancy-related pathologies. We therefore evaluated the expression level of DSCAM using real time RT-PCR in comparison to three reference genes recommended for the analysis of placental tissue (SDHA, TBP, and YWHAZ) [20]. We did not find any significant difference for DSCAM expression between placentas from normal and pathological pregnancies (idiopathic growth restriction, growth restriction of vascular origin or pre-eclampsia (Fig. 4a).

In order to decipher a possible role of DSCAM in trophoblast syncytialisation, primary human trophoblastic cells from first trimester conceptuses were isolated and grown in short-term culture $(72 \mathrm{~h})$ to spontaneously differentiate into syncytiotrophoblast. By real time RT PCR, we evaluated the level of DSCAM expression in these samples, during differentiation. The expression of DSCAM was overall low and no difference could be detected during the process of syncytialisation (Fig. 4b). In a test of in vitro syncytialisation using BeWo cells treated with Forskolin, DSCAM levels were too low to show any effect. 
Fig. 3 Expression of DSCAM in placental tissues.

a-e Immunohistochemistry on placental slides with an antiDSCAM antibody (60x magnification). The gestational age of placentas is notified in the lower right corner in weeks of amenorrhea. Scale bar $10 \mu \mathrm{m}$. ST syncytiotrophoblasts, EC endothelial cells. a, c Staining from a representative sample; b negative control, in the absence of the primary antibody; d negative control in the presence of the blocking DSCAM peptide; e control in the presence of a blocking peptide irrelevant to DSCAM (Sly peptide)
A)

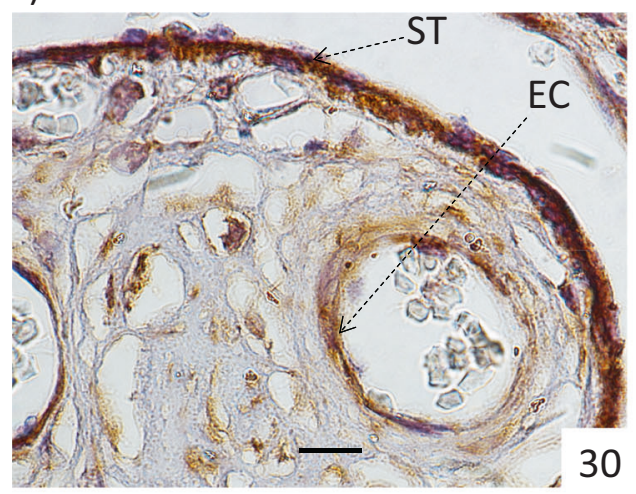

C)

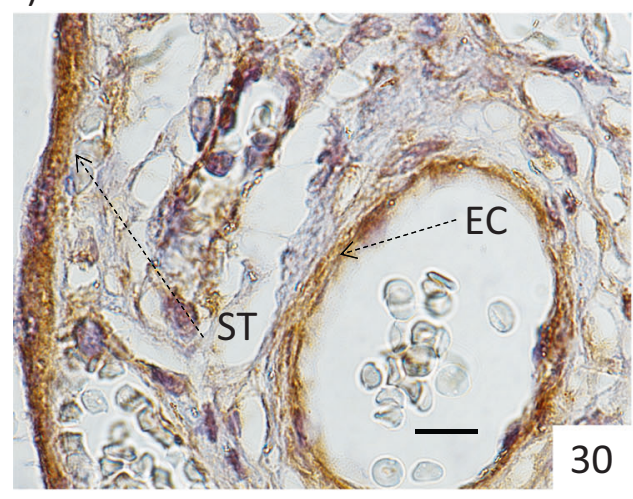

B)

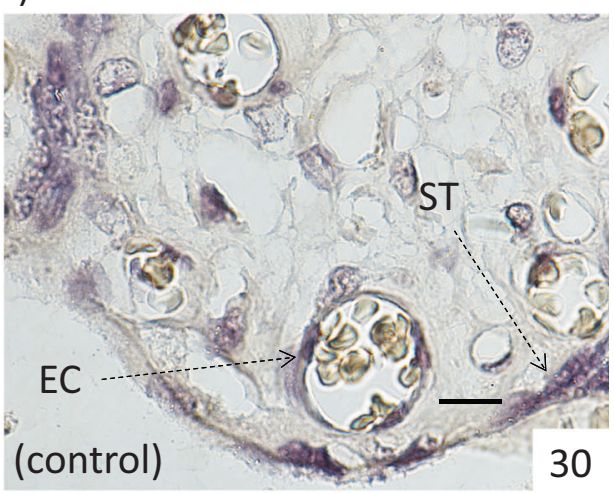

D)

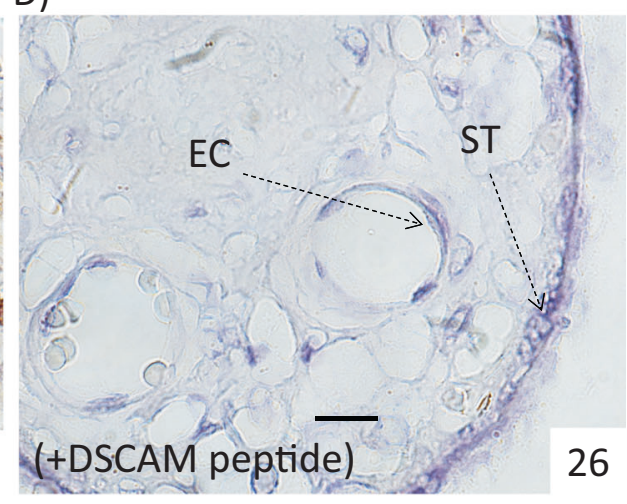

E)

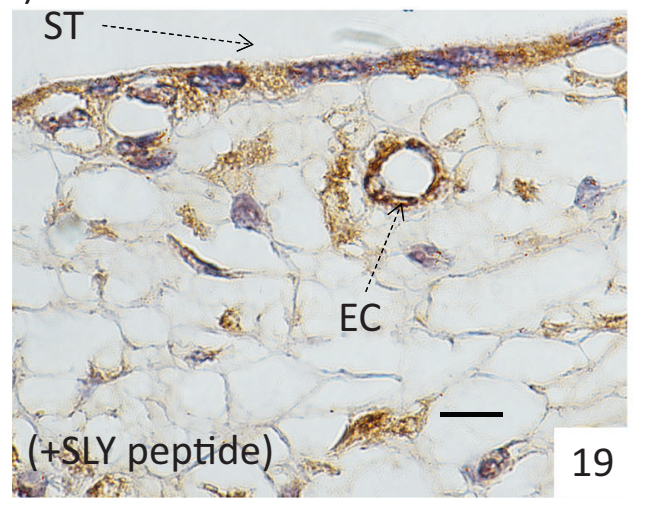

\section{Dscam imprinting status in mice}

The imprinting status of Dscam was investigated in samples obtained from reciprocal crosses between the classical C57/ B6 laboratory mouse and the Mus musculus molossinus subline. Amplifications around 3 consecutive SNPs rs46901054:A > G (NC_000082.6:g.96685216 A > G), rs46747365:A > G (NC_000082.6:g.96683850 A > G) and rs46432878:T > C (NC_000082.6:g.96685258 T > C) were performed on various cDNAs samples obtained from F1 animals identified as heterozygous for the three SNPs. Finely dissected foetal cerebral tissues were analysed because Dscam is preferentially expressed in the neuronal tissue and participates to neuron development. A biallelic expression was obtained in hindbrain, cerebellum, spinal cord, midbrain and cortex samples of three foetuses from reciprocal crosses at E13.5 and E18.5. Placentas were either kept intact or dissected to obtain separately the labyrinth and junctional zone. Expression also proved to be biallelic in all samples at E13.5, E16.5 or E18.5 $(n=10$ placental samples from five reciprocal crosses and 29 labyrinth/ junctional zone pairs from 10 reciprocal crosses). As a control, the expression of the paternally expressed Igf2 gene was checked via the coding SNP rs248081537:G > A (NC_000073.6:g.142654316 G > A) in the same samples. All placental tissues exhibited the expected strictly paternal 


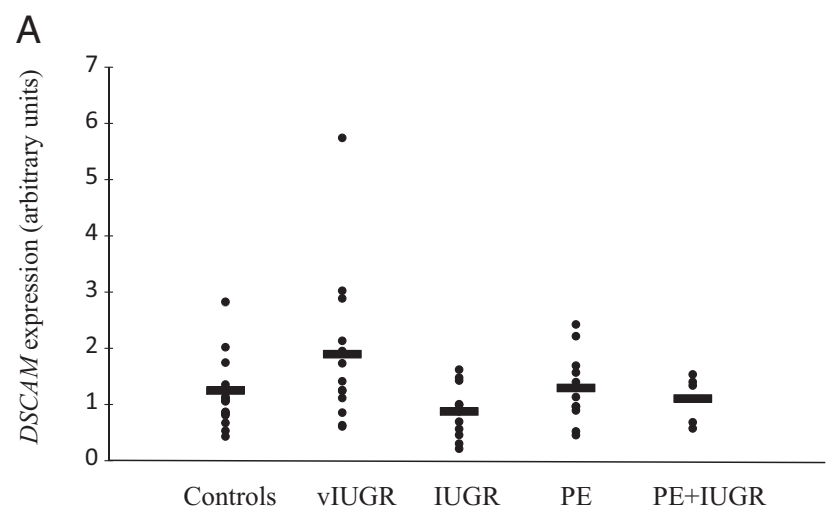

B

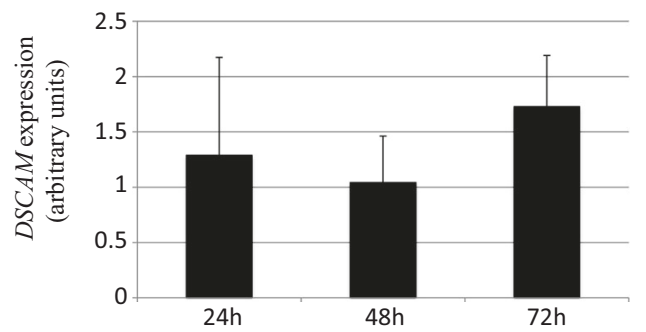

Fig. 4 DSCAM expression levels in placental samples. a Real time RTPCR in pathological and control placental samples. (IUGR intrauterine growth restriction $(n=13)$, vIUGR intrauterine growth restriction of vascular origin $(n=15)$, PE preeclampsia $(n=12)$, PE + IUGR combination of the maternal and foetal pathologies $(n=5)$; controls $(n=20))$. The expression of DSCAM was normalised using three housekeeping genes. b Real time RT-PCR in human isolated trophoblasts during spontaneous syncytialisation in vitro. $(24 \mathrm{~h}, n=7 ; 48 \mathrm{~h}$, $n=6 ; 72 \mathrm{~h}, n=4)$

expression whereas cerebral samples showed a biallelic expression (data not shown).

\section{Discussion}

Imprinted genes are a subset of genes presenting a very peculiar mode of expression that is interesting both from the fundamental point of view and in relation to human pathologies. Since the identification of the first imprinted genes in the 80 's, the list has reached about 150 in mice and 100 in human, without certitude about the real definitive number. Our goal was to identify new imprinted genes in the human placenta, particularly in relation to placental pathologies. We developed a strategy that already revealed the existence of 7 novel imprinted genes. We pursued here this work that now leads to the identification of two novel imprinted genes, AIMI and DSCAM.

The first one, $A I M 1$, has recently been described as imprinted for the first time simultaneously to our study [21]. We confirm the isoform-specific imprinting of this gene, as the long protein-coding transcript expressed from an upstream promoter is imprinted whereas the short isoform has a biallelic expression. This imprinting model has already been described for the MEST gene in human and pig [27, 28] and GNAS for example [29]. Recent data suggest that this phenomenon is rather common to many imprinted genes [30]. The NCAM2 gene also shows a monoallelic maternal expression in most studied cases but some exceptions need to be verified. Such an example of an imprinted gene of random parental origin has also been reported for the ZFAT gene [31] and in these cases, extended cohorts should be analysed to determine the exact pattern of expression of these genes.

We also identified and validated the DSCAM gene as imprinted in the human placenta and showing an exclusive expression of the paternal allele. We could not determine if DSCAM-AS1 and DSCAM-IT1, two non-coding RNAs present at the DSCAM locus were also imprinted, because of a too weak expression level in placental samples. Antisense RNAs to imprinted genes are often prone to the same regulation, as for ZFAT-ASI/ZFAT, IGF2AS/IGF2 [32], and contribute to the complex regulation of these loci. In mouse, Dscam exhibits a biallelic expression in placental and cerebral tissues, a divergence between species already observed for some imprinted genes [33].

We explored the DSCAM locus to identify a DMR that could control its monoallelic expression. A genome-wide methylation study by Fang et al. orientated us towards a region located in intron 1 [25] that we confirmed as an imprinted DMR in placenta. To explore the parent-of-origin of the methylated and unmethylated alleles, we genotyped the only SNPs reported in this region but failed to identify any heterozygous among placenta samples because of the rareness of the variants. Therefore, we decided to address this question indirectly by assessing the methylation level of this region in sperm cells. Sperm DNA analyses showed a very weak methylation level, compatible with that observed by Fang et al. [25]. We concluded that this intron 1 DMR is maternally imprinted, compatible with a paternal expression of the DSCAM gene. We could identify in this region (www.genomatix.de) two putative binding sites for the well-known insulator CTCF as well as for ZAC1/PLAGL1, the coordinator of the mouse imprinted gene network [34] that could relate DSCAM to other imprinted genes.

The DSCAM gene encodes a cellular adhesion molecule from the immunoglobulin family known to play roles in neural development and function in mammals and insects. In Drosophila, this gene exhibits a particularly rich alternative splicing producing tens of thousands of isoforms [35] while in Arthropods, it is known for its immunologic function [36]. DSCAM has been shown to be involved in axon guidance, dendrite arborisation involving selfavoidance and tiling [37]. Placentas are devoid of neurons and our investigations concluded that DSCAM was present in endothelial cells and syncytiotrophoblasts in the human 
placenta. Expression studies and knock-out experiment in Zebrafish tend to suggest that Dscam plays a role in cellular differentiation in many cell types [38]. Therefore, a new function for DSCAM needs to be found in placental tissue.

DSCAM is a receptor for the secreted guidance factor Netrin 1 [39], which also binds to other receptors such as Neogenin, Deleted in Colorectal Cancer (DCC) and Uncoordinated-5 homologue (UNC5B). These genes have been extensively studied in the central nervous system but they are also expressed in the placenta [40, 41]. DCC, similarly to DSCAM, is expressed by syncytiotrophoblasts, while Netrin 1 is detected in early cytotrophoblasts and later outside syncytiotrophoblasts. Interestingly, placentas from Down syndrome patients present an abnormal development of the syncytiotrophoblast layer [42]. It would be interesting to investigate whether this is due to a deregulation of DSCAM expression or function. We did not dispose of Trisomy 21 placentas to address this question. However, we evaluated DSCAM expression levels in our collection of human placenta samples from normal and pathological pregnancies (IUGR, pre-eclampsia) and the differences did not reach statistical significance though the expression level of Netrin 1, DSCAM ligand, has been shown to be reduced in placentas from foetal growth restricted babies [43].

Mice carrying homozygous deletion or mutation of the Dscam gene generally pursue their development until birth, with variations in the phenotype and in its severity according to backgrounds and responsible mutations. Some die shortly after birth from respiratory distress due to an abnormal set up of neurons controlling the diaphragm [44]. This shows that Dscam is not strictly compulsory to intrauterine life. Its absence can be compatible with placental and foetal development. Many other imprinted gene knockout mice produce viable progeny as well, such as Igf2, H19, etc [45, 46]. Dscam null mice phenotype was mainly studied in a neuro-developmental context, including abnormal retinal development, abnormal amacrine cell morphology [44, 47, 48]. Effects on reproduction were not detailed though a delay of gestation was observed [44] as well as an abnormal maternal behaviour [47].

The localisation of this gene on chromosome 21 is a novelty as no imprinted gene had been assigned to the smallest human autosome so far. Though we specifically targeted our previous results on this chromosome after the positive result of DSCAM and analysed some additional candidates, we have not been able to identify any other imprinted gene on chromosome 21 except an inconclusive status for NCAM2 that needs to be confirmed on a larger cohort. Stoll et al. had suggested that this chromosome was devoid of imprinted genes as they could not find any evidence of phenotypic difference between Down syndrome patients having an extra chromosome 21 of either paternal or maternal origin [49]. However, the phenotypic heterogeneity of the disease makes correlations difficult [50]. The non-disjunction error responsible for the disease is very frequently of maternal origin $(90 \%)$ [51]. In these cases, the level of a paternally expressed gene such as DSCAM would not be affected by the presence of the supernumerary chromosome. DSCAM has been proposed as a candidate gene responsible for intellectual disability in Down syndrome [52], and for cardiac and visceral malformations [53, 54].

In sum, we identified DSCAM as a new imprinted gene in the human placenta. Its role and function in this tissue is still unclear and additional studies are needed to decipher the role it may play in placental tissue and how it could interact with other imprinted genes within the IG Network. Given the limited data available, we hypothesise that DSCAM could promote placental growth. Indeed, according to the theory of conflict, imprinted genes expressed from the paternal copy, as DSCAM, tend to promote growth of the foeto-placental unit.

\section{Deposited data}

Imprinting results for DSCAM, AIM1 and NCAM2 have been deposited in the LOVD database under the references DSCAM_000004, AIM1_000006 and NCAM2_000001, respectively.

Acknowledgements We are indebted to Dr Ahmed Ziyyat and Brigitte Lefèvre for their help. We are thankful to all patients and medical staff participating to the Biogestelle collection at the Cochin Hospital. We are grateful to Dr Deborah Bourc'his, Juliane Glaser, Raquel Perez Palacios, Guillaume Pidoux, Elma El Khouri, Charlotte Moretti, Côme Ialy-Radio, Béatrice Parfait and Paul Monnier for their help. The platforms of the Cochin Institute, Imagerie Cellulaire and GenomIC are thanked for their help and support. This work was supported by the ANR TWOTHYME.

\section{Compliance with ethical standards}

Conflict of interest The authors declare that they have no conflict of interest.

\section{References}

1. McGrath J, Solter D. Completion of mouse embryogenesis requires both the maternal and paternal genomes. Cell. 1984;37:179-83. Epub 1984/05/01

2. Tycko B. Imprinted genes in placental growth and obstetric disorders. Cytogenet Genome Res. 2006;113:271-8. Epub 2006/04/01

3. Monk D. Genomic imprinting in the human placenta. Am J Obstet Gynecol. 2015;213(4 Suppl):S152-62. Epub 2015/10/03

4. Hanna CW, Penaherrera MS, Saadeh H, et al. Pervasive polymorphic imprinted methylation in the human placenta. Genome Res. 2016;26:756-67. Epub 2016/01/16

5. Keverne B. Monoallelic gene expression and mammalian evolution. Bioessays. 2009;31:1318-26. Epub 2009/11/19 
6. Angiolini E, Fowden A, Coan P, et al. Regulation of placental efficiency for nutrient transport by imprinted genes. Placenta. 2006;27(Suppl A):S98-102. Epub 2006/03/01

7. Sittig LJ, Redei EE. Fine-tuning notes in the behavioral symphony: parent-of-origin allelic gene expression in the brain. Adv Genet. 2014;86:93-106. Epub 2014/08/31

8. Calabrese JM, Starmer J, Schertzer MD, Yee D, Magnuson T. A survey of imprinted gene expression in mouse trophoblast stem cells. G3 (Bethesda). 2015;5:751-9. Epub 2015/02/26

9. Yuen RK, Jiang R, Penaherrera MS, McFadden DE, Robinson WP. Genome-wide mapping of imprinted differentially methylated regions by DNA methylation profiling of human placentas from triploidies. Epigenetics Chromatin. 2011;4:10. Epub 2011/ $07 / 14$

10. Sanchez-Delgado M, Court F, Vidal E, et al. Human oocytederived methylation differences persist in the placenta revealing widespread transient imprinting. PLoS Genet. 2016;12:e1006427. Epub2016/11/12

11. Hamada $\mathrm{H}$, Okae $\mathrm{H}$, Toh $\mathrm{H}$, et al. Allele-specific methylome and transcriptome analysis reveals widespread imprinting in the human placenta. Am J Hum Genet. 2016;99:1045-58. Epub 2016/ $11 / 16$

12. Choi NY, Bang JS, Lee HJ, et al. Novel imprinted single CpG sites found by global DNA methylation analysis in human parthenogenetic induced pluripotent stem cells. Epigenetics. 2018;13(4):343-51. Epub 2018/04/04.

13. Luedi PP, Dietrich FS, Weidman JR, et al. Computational and experimental identification of novel human imprinted genes. Genome Res. 2007;17:1723-30. Epub 2007/12/07

14. Luedi PP, Hartemink AJ, Jirtle RL. Genome-wide prediction of imprinted murine genes. Genome Res. 2005;15:875-84. Epub 2005/06/03

15. Barbaux S, Gascoin-Lachambre G, Buffat C, et al. A genomewide approach reveals novel imprinted genes expressed in the human placenta. Epigenetics. 2012;7:1079-90. Epub 2012/08/17

16. Miller SA, Dykes DD, Polesky HF. A simple salting out procedure for extracting DNA from human nucleated cells. Nucleic Acids Res. 1988;16:1215. Epub 1988/02/11

17. Gascoin-Lachambre G, Buffat C, Rebourcet R, et al. Cullins in human intra-uterine growth restriction: expressional and epigenetic alterations. Placenta. 2010;31:151-7. Epub 2009/12/17

18. Benaitreau D, Dos Santos E, Leneveu MC, et al. Adiponectin promotes syncytialisation of BeWo cell line and primary trophoblast cells. Reprod Biol Endocrinol. 2010;8:128. Epub 2010/11/03

19. Poidatz D, Dos Santos E, Brule A, De Mazancourt P, Dieudonne MN. Estrogen-related receptor gamma modulates energy metabolism target genes in human trophoblast. Placenta. 2012;33:68895. Epub 2012/07/06

20. Meller M, Vadachkoria S, Luthy DA, Williams MA. Evaluation of housekeeping genes in placental comparative expression studies. Placenta. 2005;26:601-7. Epub 2005/08/09

21. Das R, Lee YK, Strogantsev R, et al. DNMT1 and AIM1 Imprinting in human placenta revealed through a genome-wide screen for allele-specific DNA methylation. BMC Genom. 2013;14:685. Epub 2013/10/08

22. Nelson EA, Walker SR, Li W, Liu XS, Frank DA. Identification of human STAT5-dependent gene regulatory elements based on interspecies homology. J Biol Chem. 2006;281:26216-24. Epub 2006/07/15

23. Lambertini L, Diplas AI, Lee MJ, et al. A sensitive functional assay reveals frequent loss of genomic imprinting in human placenta. Epigenetics. 2008;3:261-9. Epub 2008/09/05

24. Agarwala KL, Ganesh S, Amano K, Suzuki T, Yamakawa K. DSCAM, a highly conserved gene in mammals, expressed in differentiating mouse brain. Biochem Biophys Res Commun. 2001;281:697-705. Epub 2001/03/10
25. Fang F, Hodges E, Molaro A, et al. Genomic landscape of human allele-specific DNA methylation. Proc Natl Acad Sci USA. 2012;109:7332-7. Epub 2012/04/24

26. Docherty LE, Rezwan FI, Poole RL, et al. Genome-wide DNA methylation analysis of patients with imprinting disorders identifies differentially methylated regions associated with novel candidate imprinted genes. J Med Genet. 2014;51:229-38. Epub 2014/02/07

27. Li X, Song N, Wang D, et al. Isoform-specific imprinting of the MEST gene in porcine parthenogenetic fetuses. Gene. 2015;558:287-90. Epub 2015/01/20

28. Pedersen IS, Dervan P, McGoldrick A, et al. Promoter switch: a novel mechanism causing biallelic PEG1/MEST expression in invasive breast cancer. Hum Mol Genet. 2002;11:1449-53. Epub $2002 / 05 / 25$

29. Peters J, Holmes R, Monk D, et al. Imprinting control within the compact Gnas locus. Cytogenet Genome Res. 2006;113:194-201. Epub 2006/04/01

30. Stelzer Y, Bar S, Bartok O, et al. Differentiation of human parthenogenetic pluripotent stem cells reveals multiple tissue- and isoform-specific imprinted transcripts. Cell Rep. 2015;11:308-20. Epub 2015/04/07

31. Metsalu T, Viltrop T, Tiirats A, et al. Using RNA sequencing for identifying gene imprinting and random monoallelic expression in human placenta. Epigenetics. 2014;9:1397-409. Epub 2014/12/02

32. Kanduri C. Long noncoding RNAs: lessons from genomic imprinting. Biochim Biophys Acta. 2016;1859(1):102-11. Epub 2015/05/26.

33. Rademacher K, Schroder C, Kanber D, et al. Evolutionary origin and methylation status of human intronic $\mathrm{CpG}$ islands that are not present in mouse. Genome Biol Evol. 2014;6:1579-88. Epub 2014/06/14

34. Varrault A, Gueydan C, Delalbre A, et al. Zac1 regulates an imprinted gene network critically involved in the control of embryonic growth. Dev Cell. 2006;11:711-22. Epub 2006/11/07

35. Zipursky SL, Wojtowicz WM, Hattori D. Got diversity? Wiring the fly brain with Dscam. Trends Biochem Sci. 2006;31:581-8. Epub 2006/08/22

36. Schmucker D, Chen B. Dscam and DSCAM: complex genes in simple animals, complex animals yet simple genes. Genes Dev. 2009;23:147-56. Epub 2009/01/28

37. Millard SS, Zipursky SL. Dscam-mediated repulsion controls tiling and self-avoidance. Curr Opin Neurobiol. 2008;18:84-9. Epub 2008/06/10

38. Yimlamai D, Konnikova L, Moss LG, Jay DG. The zebrafish down syndrome cell adhesion molecule is involved in cell movement during embryogenesis. Dev Biol. 2005;279:44-57. Epub 2005/02/15

39. Liu G, Li W, Wang L, et al. DSCAM functions as a netrin receptor in commissural axon pathfinding. Proc Natl Acad Sci USA. 2009;106:2951-6. Epub 2009/02/07

40. Dakouane-Giudicelli M, Duboucher C, Fortemps J, et al. Characterization and expression of netrin- 1 and its receptors UNC5B and DCC in human placenta. $\mathrm{J}$ Histochem Cytochem. 2010;58:73-82. Epub 2009/10/15

41. Dakouane-Giudicelli M, Duboucher C, Fortemps J, et al. Identification and localization of netrin-4 and neogenin in human first trimester and term placenta. Placenta. 2012;33:677-81. Epub 2012/06/19

42. Malassine A, Pidoux G, Gerbaud P, Frendo JL, Evain-Brion D. Human trophoblast in trisomy 21: a model for cell-cell fusion dynamic investigation. Adv Exp Med Biol. 2011;714:103-12. Epub 2011/04/21

43. Qian-hua W, Shao-ping Z, Jian-wen Z, Yun Y, Li Z. Reduced expression of netrin-1 is associated with fetal growth restriction. Mol Cell Biochem. 2011;350:81-7. Epub 2011/01/05 
44. Amano K, Fujii M, Arata S, et al. DSCAM deficiency causes loss of pre-inspiratory neuron synchroneity and perinatal death. The Journal Neurosci. 2009;29:2984-96. Epub 2009/03/06

45. Ripoche MA, Kress C, Poirier F, Dandolo L. Deletion of the H19 transcription unit reveals the existence of a putative imprinting control element. Genes Dev. 1997;11:1596-604. Epub 1997/06/15

46. Varmuza S, Miri K. What does genetics tell us about imprinting and the placenta connection? Cell Mol life Sci. 2015;72:51-72. Epub 2014/09/10

47. Fuerst PG, Koizumi A, Masland RH, Burgess RW. Neurite arborization and mosaic spacing in the mouse retina require DSCAM. Nature. 2008;451:470-4. Epub 2008/01/25

48. Schramm RD, Li S, Harris BS, et al. A novel mouse Dscam mutation inhibits localization and shedding of DSCAM. PLOS ONE. 2012;7:e52652. Epub2013/01/10

49. Stoll C, Alembik Y, Dott B, Feingold J. No evidence for genomic imprinting in liver-born Down syndrome patients. Acta Genet Med Gemellol (Roma). 1996;45:265-71. Epub 1996/01/01
50. Muranjan M, Chaudhari T, Vundinti BR. Phenotypic heterogeneity and parental origin of extra chromosome 21 in Down syndrome. Indian Pediatr. 2010;47:429-32. Epub 2009/08/13

51. Ballesta F, Queralt R, Gomez D, et al. Parental origin and meiotic stage of non-disjunction in 139 cases of trisomy 21. Ann Genet. 1999;42:11-5. Epub 1999/04/24

52. Yamakawa K, Huot YK, Haendelt MA, et al. DSCAM: a novel member of the immunoglobulin superfamily maps in a Down syndrome region and is involved in the development of the nervous system. Hum Mol Genet. 1998;7:227-37. Epub 1998/ $03 / 21$

53. Jannot AS, Pelet A, Henrion-Caude A, et al. Chromosome 21 scan in Down syndrome reveals DSCAM as a predisposing locus in Hirschsprung disease. PLoS ONE. 2013;8:e62519. Epub2013/05/15

54. Barlow GM, Chen XN, Shi ZY, et al. Down syndrome congenital heart disease: a narrowed region and a candidate gene. Genet Med. 2001;3:91-101. Epub 2001/04/03 